\title{
Coronary Artery Bypass Grafting Complicated by Post-operative Coronavirus Infection - Two Similar Presentations with Dissimilar Outcomes
}

\author{
Siddharth Pahwa ${ }^{1}$, Harideep Samanapally ${ }^{2}$, Mike O. Udoh ${ }^{3}$, Jiapeng Huang ${ }^{3}$, and Mark \\ Slaughter ${ }^{1}$ \\ ${ }^{1}$ University of Louisville Division of Thoracic and Cardiovascular Surgery \\ ${ }^{2}$ University of Louisville Division of Infectious Diseases \\ ${ }^{3}$ University of Louisville
}

November 8, 2021

\begin{abstract}
Patients diagnosed with COVID-19 infection undergoing surgical procedures have been reported to have increased post-operative complications and mortality. These findings are important when considering cardiac surgical procedures, specifically coronary artery bypass grafting (CABG). This case series describes the clinical course following a CABG procedure in two patients that went on to develop COVID-19 infection post-operatively, having previously tested negative. We aim to illustrate the similarities in clinical presentation, but differences in eventual outcomes for both patients and hypothesize the reasons for the differences. Patients with comorbidities such as advanced age, diabetes mellitus, obesity, hypertension, and COPD are possibly at increased risk of adverse outcomes should they contract the infection, and special care should be taken in this population. Early institution of VV-ECMO may be beneficial, but further studies are needed in this matter.
\end{abstract}

\section{Introduction}

The Coronavirus Disease 2019 (COVID-19) pandemic continues to complicate surgical care delivery for patients suffering from cardiovascular disease. Additionally, the development of virulent coronavirus strains increases the need for literature on post-operative complications associated with COVID-19. Patients diagnosed with COVID-19 infection undergoing surgical procedures have been reported to have increased postoperative complications and mortality. ${ }^{1-3}$ Knisely et al reported increased morbidity and mortality in patients who underwent urgent surgical procedures and contracted COVID-19 either pre- or post-operatively. ${ }^{4}$ These findings are important when considering cardiac surgical procedures, specifically coronary artery bypass grafting (CABG) during this pandemic, since the Society of Thoracic Surgeons (STS) describes most of these operations as 'urgent'. ${ }^{5}$ In addition, the majority of cardiac surgical patients are at increased risk of infection and death with COVID-19, as they are frequently of old age, obese, hypertensive, and diabetic, with severe cardiac or pulmonary diseases. ${ }^{6}$

This case series describes the clinical course following a CABG procedure in two patients that went on to develop COVID-19 infection post-operatively. We aim to illustrate the similarities in clinical presentation, but differences in eventual outcomes for both patients and hypothesize the reasons for the differences. Institutional review board approval was waived since this was a minimal risk, case se

\section{Case series}

Patient 1: A 42-year-old, hypertensive gentleman presented with unstable angina and was diagnosed with triple vessel coronary artery disease by left heart catheterization. His initial hemodynamics and labs were 
within normal limits and are described in Table 1. Pre-operative arterial blood gas (ABG) analysis was normal on room air (Table 2). Patient tested negative for COVID-19 by reverse transcription polymerase chain reaction (RT-PCR) one day prior to the CABG procedure.

The patient underwent on-pump CABG x 3 (left internal mammary artery to the left anterior descending artery, sequential saphenous vein graft to the posterior descending and obtuse marginal arteries). The surgery was carried out uneventfully and the intra-operative ABG on a fraction of inspired oxygen (FiO2) of $70 \%$ is depicted in Table 2. The patient was weaned off cardio-pulmonary bypass on no inotropes, and a small dose of vasopressor. In the intensive care unit, the first ABG on a $\mathrm{FiO} 2$ of $100 \%$ was abnormal with a partial pressure of oxygen (PO2) of $69 \mathrm{mmHg}$ (Table 2). A few hours after surgery, the patient became hypotensive with low cardiac indices. An epinephrine infusion was started, and vasopressors were escalated. The patient was initially dyssynchronous on the ventilator and needed deepened sedation. He continued to have low arterial saturations on escalating ventilatory support over the next $24-48$ hours. A repeat COVID-19 RT-PCR was sent due to the ongoing difficulty with ventilation, which returned positive on post-operative day (POD)-2.

On POD-3, the patient was placed on peripheral veno-venous extracorporeal membrane oxygenation (VVECMO). He responded well on ECMO with improved arterial saturations. The ECMO run was complicated by positive blood cultures, for which antibiotics were escalated. A percutaneous tracheostomy was performed on POD-15. After being supported on VV-ECMO for 18 days, the patient was successfully weaned off and decannulated. He was then slowly weaned off the ventilator and after being on the tracheostomy for 22 days, he was then decannulated and was discharged home.

Patient 2: A 62-year-old morbidly obese lady with sleep apnea, type-2 diabetes mellitus, chronic obstructive pulmonary disease on home oxygen, and hypertension presented to the hospital with non-ST elevation myocardial infarction. Her initial hemodynamics and labs were within normal limits (Table 1) except a random blood sugar of $322 \mathrm{mg} / \mathrm{dl}$, with a HbA1c of $9.2 \%$. Coronary angiography revealed critical threevessel disease. Her chest x-ray revealed bronchopneumonia of right lower lobe, for which antibiotics were initiated. Her pre-operative ABG on 3 liters of oxygen/minute is depicted in Table 2. She tested negative for COVID-19 six days prior to surgery by RT-PCR.

After medical optimization, she underwent on-pump CABG x 3 (left internal mammary artery to the left anterior descending artery and individual reverse saphenous vein grafts to the obtuse marginal and right coronary arteries). The surgery was carried out uneventfully, and she left the operating room on no inotropes or vasopressors. Post-operatively, she developed hypoxia with escalating PEEP and FiO2 requirements (Table 2). Her cardiac indices were borderline low, and she was started on an epinephrine and milrinone infusion. The ventilatory struggles continued for the next 48-72 hours, with the patient being dyssynchronous on the ventilator, responding marginally to ketamine infusion. On POD-6, RT-PCR for COVID-19 returned positive, and she was started on remdesivir. There was marginal improvement in oxygenation, and she was supported on the ventilator for another 7 days. On POD-14, she was placed on peripheral VV-ECMO. She initially responded well to ECMO, but later developed heparin-induced thrombocytopenia (HIT). She also had intermittent fevers, with escalating antibiotic requirements. A percutaneous tracheostomy was also performed. However, she showed improvement from a respiratory standpoint, and after 12 days on ECMO support, she was weaned off and decannulated. The patient then remained stable for 24-48 hours, but then started developing significantly elevated liver function markers with abdominal distention. A rising serum lactate levels led to the suspicion of bowel ischemia, and an exploratory laparotomy was performed. The laparotomy revealed extensive small bowel ischemia, and given the extent of the disease, a decision was reached with the family to make her comfort care.

\section{Discussion}

The COVID-19 pandemic continues to ravage surgical care of critically ill patients. Patients undergoing CABG, who develop COVID-19 infection in the peri-operative period, are at an increased risk of morbidities and mortality, with prolonged hospital stay. ${ }^{7}$ In our series, both patients had respiratory failure, needing VV- 
ECMO support with long hospital stays. However, the outcome was worse for the patient with pre-existing comorbidities of morbid obesity, diabetes mellitus, hypertension, and COPD.

Since the beginning of the pandemic, hospitals have been brainstorming to design protocols to test asymptomatic patients being admitted for 'routine' cardiac surgeries. Initially, the testing was limited to the high-risk population group, but very soon it was extended to pre-operatively screen all patients undergoing surgery. In our case, both patients had pre-operative negative COVID-19 tests but tested positive soon after surgery. Whether the initial result was false-negative ${ }^{8}$ and the patients contracted the virus pre-operatively, either prior to admission or in the hospital, is difficult to ascertain. The clinical manifestations, however, only appeared in the early post-operative period. Evidence suggests that CPB activates inflammatory responses which can lead to lung tissue damage as well as increased pulmonary endothelial permeability. ${ }^{9}$ The cumulative effect of inflammation from CABG and COVID-19 is a possible source for the morbidity and mortality in patients with COVID-19 infection peri-operatively.

Both patients had similar clinical presentations, including ventilatory dyssynchrony, in the immediate postoperative period, leading to escalating ventilator parameters and sedation requirements. Veno-venous ECMO was initiated early on the first patient, one day after the diagnosis of COVID-19, whereas it was initiated eight days after diagnosis on the second patient. The role of early institution of VV-ECMO in severe COVID-19 infection is being investigated ${ }^{10}$, specially in post cardiotomy patients.

Cardiac surgery volumes have dramatically reduced across the nation during the pandemic, with an even more dramatic increase in operative mortality. ${ }^{5}$ In order to safely perform 'routine, low-risk' cardiac surgeries, one must have robust and effective pre-surgery screening protocols, with a low threshold to test patients again post-operatively should it be needed clinically. Patients with comorbidities such as advanced age, diabetes mellitus, obesity, hypertension, and COPD are possibly at increased risk of adverse outcomes should they contract COVID-19, and special care should be taken in this population. Early institution of VV-ECMO may be beneficial, but further studies are needed in this matter.

\section{References}

1. Abate SM, Mantefardo B, Basu B. Postoperative mortality among surgical patients with COVID-19: a systematic review and meta-analysis. Patient Saf Surg. 2020 Oct 12; 14:37.

2. Søreide K, Hallet J, Matthews JB, Schnitzbauer AA, Line PD, Lai PBS, et al. Immediate and long-term impact of the COVID-19 pandemic on delivery of surgical services. Br J Surg. 2020 Sep;107(10):12501261.

3. Aziz H, Filkins A, Kwon YK. Review of COVID-19 Outcomes in Surgical Patients. Am Surg. 2020 Jul;86(7):741-745.

4. Knisely, A., Zhou, Z. N., Wu, J., Huang, Y., Holcomb, K., Melamed, A., et al. Perioperative Morbidity and Mortality of Patients With COVID-19 Who Undergo Urgent and Emergent Surgical Procedures (2021). Annals of surgery, 273(1), 34-40.

5. Nguyen TC, Thourani VH, Nissen AP, Habib RH, Dearani JA, Ropski A, et al. The Effect of COVID19 on Adult Cardiac Surgery in the United States in 717103 Patients. Ann Thorac Surg. 2021 Jul 31: S0003-4975(21)01310-2.

6. Huang C, Wang Y, Li X, Ren L, Zhao J, Hu Y, et al. Clinical features of patients infected with 2019 novel coronavirus in Wuhan, China. Lancet. 2020 Feb 15;395(10223):497-506.

7. Yates MT, Balmforth D, Lopez-Marco A, Uppal R, Oo AY. Outcomes of patients diagnosed with COVID-19 in the early postoperative period following cardiac surgery. Interact Cardiovasc Thorac Surg. 2020 Oct 1;31(4):483-485.

8. Arevalo-Rodriguez, I., Buitrago-Garcia, D., Simancas-Racines, D., Zambrano-Achig, P., Del Campo, R., Ciapponi, A., et al (2020). False-negative results of initial RT-PCR assays for COVID-19: A systematic review. PloS one, 15(12), e0242958.

9. Montes FR, Maldonado JD, Paez S, Ariza F. Off-pump versus on-pump coronary artery bypass surgery and postoperative pulmonary dysfunction. J Cardiothorac Vasc Anesth. 2004 Dec;18(6):698-703.

10. Mustafa AK, Alexander PJ, Joshi DJ, Tabachnick DR, Cross CA, Pappas PS, et al. Extracorporeal 
Membrane Oxygenation for Patients With COVID-19 in Severe Respiratory Failure. JAMA Surg. 2020 Oct 1;155(10):990-992.

Table 1 - Patient Characteristics

\begin{tabular}{|c|c|c|}
\hline Characteristics & Patient 1 & Patient 2 \\
\hline Age (years) & 42 & 62 \\
\hline Sex & Male & Female \\
\hline Clinical presentation & Unstable angina & $\begin{array}{l}\text { Non-ST elevation myocardial } \\
\text { infarction }\end{array}$ \\
\hline Left heart catheterization & Three vessel disease & Three vessel disease \\
\hline Co-morbidities & Hypertension & $\begin{array}{l}\text { Hypertension Type } 2 \text { Diabetes } \\
\text { mellitus Morbid obesity Chronic } \\
\text { obstructive pulmonary disease on } \\
\text { home oxygen Obstructive sleep } \\
\text { apnea }\end{array}$ \\
\hline Serum creatinine $(\mathrm{mg} / \mathrm{dl})$ & 0.86 & 1.1 \\
\hline $\begin{array}{l}\text { Pre-operative Carotid duplex } \\
\text { scan }\end{array}$ & $\begin{array}{l}<50 \% \text { stenosis of bilateral } \\
\text { internal carotid arteries }\end{array}$ & $\begin{array}{l}>90 \% \text { stenosis of left internal } \\
\text { carotid artery; } 50-69 \% \text { stenosis } \\
\text { of right internal carotid artery }\end{array}$ \\
\hline STS risk of mortality & $0.9 \%$ & $2.7 \%$ \\
\hline Pre-CPB TEE & Normal valves, LVEF $50 \%$ & Normal valves, LVEF $50 \%$ \\
\hline Post-CPB TEE & Normal valves, LVEF $55-60 \%$ & Normal valves, LVEF $50 \%$ \\
\hline Positive COVID-19 RT-PCR & Post-operative day 2 & Post-operative day 6 \\
\hline Days on V-V ECMO & 18 & 12 \\
\hline Length of hospital stay (days) & 54 & 36 \\
\hline $\begin{array}{l}\text { Other post-operative } \\
\text { complications }\end{array}$ & Septicemia & $\begin{array}{l}\text { Septicemia Clostridium difficile } \\
\text { infection Heparin-induced } \\
\text { thrombocytopenia Mesenteric } \\
\text { ischemia }\end{array}$ \\
\hline Clinical outcome & Recovered & Deceased \\
\hline
\end{tabular}

Table 2 - Peri-operative Arterial Blood Gas Analysis

\begin{tabular}{lll}
\hline ABG analysis & Patient 1 & Patient 2 \\
\hline Pre-operative & On room air $\mathrm{pH} 7.412 \mathrm{PCO} 238.1$ & On 3 liters/min O2 pH 7.295 \\
& mmHg PO2 78.6 mmHg & PCO2 53.1 mmHg PO2 103 \\
& FiO2 70\% pH 7.32 PCO2 49.1 & FiO2 70\% pH 7.281 PCO2 50.9 \\
Intra-operative & mmHg PO2 66.7 mmHg & mmHg PO2 57 mmHg \\
& FiO2 100\%, PEEP 10 mmHg pH & FiO2 100\%, PEEP 10 mmHg pH \\
Post-operative in ICU & $7.42 \mathrm{PCO} 40 \mathrm{mmHg}$ PO2 68.8 & $7.335 \mathrm{PCO} 240.4 \mathrm{mmHg}$ PO2 63 \\
& $\mathrm{mmHg}$ & $\mathrm{mmHg}$ \\
\hline
\end{tabular}

